\title{
Configuration Bitstream Mapping with Programmable Resources on Spartan-3A FPGA using XDL and FAR
}

\author{
Pravin N. Matte ${ }^{\# 1}$, Dr. Dilip D. Shah ${ }^{* 2}$ \\ \# Department of Electronics and Telecommunication, \\ G.H. Raisoni College of Engineering and Management, Wagholi, Pune, India \\ *Department of Electronics and Telecommunication Engineering, \\ JSPMs Imperial College of Engineering and Research, Wagholi, Pune, India. \\ 11 pravin.matte@raisoni.net; 2dilip.d.shah@gmail.com
}

\begin{abstract}
Configuration bitstream in FPGA is specific to vendor. It is difficult to understand bitstream format and eventually impossible to know how bits are placed in LUTs, flip flop, multiplexer, input and output interconnection network in FPGA. Normally user is not interested in knowing internals of FPGA, however it is believed that by opening the internals of FPGA, development would become very fast as it is achieved by open source community. In this paper we have proposed a simplified methodology to map configuration bitstream to its programmable resources targeting Spartan-3A FPGA. A simple AND gate design implemented in VHDL under different constraints. Correlation between different locations of AND gate is established with frame address register, XDL file report, FPGA editor and device view in PlanAhead.
\end{abstract}

Keyword - Bitstream, Configuration memory, FPGA, FAR, NCD, XDL

\section{INTRODUCTION}

It is widely believed that the analysis of bitstream format is a tedious task. Increase in the complexity of digital circuit design demand for FPGA with more resources is increasing. FPGA has applications in wide variety of domains like space, telecommunications, networking, handheld devices etc. FPGA based industries demand CAD tools for design and development to meet time-to-market design metric. Like in open source communities, FPGAs development techniques are not open and certain things are vendor specific. Hence third party tools development has limited scope. If bitstream format would have been opened to users then possibility of cloning the device and IPs would have increased. Development on design tool side demands FPGAs internal details. Knowing FPGA bitstream definitely helps further development. Beside this, research communities and academicians are interested in knowing bitstream details. Limited work has been done in this direction.

A bitstream is a file that contains programming information for an FPGA. FPGAs bitstream is generated by CAD tools and configures (reconfigures) resources on FPGA fabrics to implement desired design. Fig. 1 depicts general structure of bitstream file during configuration. Bitstream file is viewed as file containing header for device name, architecture, and date information. After the start header it has synchronization word, device identification along with various device initialization commands. Most of the data is for actual configuration of FPGA. Last part of bitstream again contains command registers like CRC, DESYNC etc. and then FPGA goes into start sequence operation. These details are contains in bitstream file. Each FPGA device has its own bitstream format to configure itself.

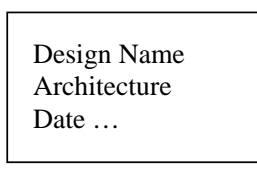

Header

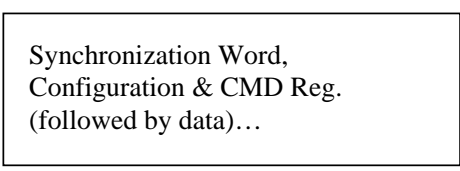

Configuration Commands

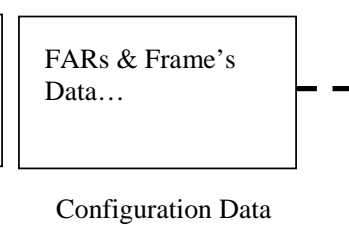

Configuration Data

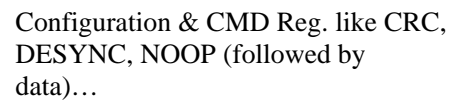
DESYNC, NOOP (followed by data)...

Configuration Commands

Fig. 1 General structure of bitstream file

Though fair amount of work is done to know bitstream format, this paper presents simple approach to understand FPGA bitstream and its mapping with resources on FPGA. Section II focused on related work done. FPGA generic architecture, design flow and bitstream format is described in section III. Preparation of background information is presented in section IV. A simple approach to analyze FPGA bitstream is elaborated in section V targeting Spartan-3A Xilinx FPGA. Results are discussed in section VI. At the end paper is concluded with conclusion in section VII. 


\section{RELATED WORK}

FPGAs configuration bitstream is the final output of any VLSI CAD tool design methodology. In [1] database of mapping bitstream to their functional role is prepared using theoretical algorithm. This database is utilized to produce net list from any bitstream. The work shown in [1] is for Xilinx Virtex-II devices. Documentation on Xilinx Design Language (XDL) is scare. XDL provide access to almost all features of Xilinx devices. Various use cases and elaborated documentation provided in [2]. In [3], a method is presented to generate NCD from bitstream file.

Due to availability of official documents on internal architecture and configuration, it was possible for researchers to build development tools for FPGA. Alex and Clifford reversed engineered Lattice Semiconductor's iCE40 FPGA. This was first step towards the source tool chain for single FPGA [4]. "Bil” a reverse engineering tool presented in [5] retrieves netlist from bitstream for certain section of bitstream. VPR [6] performs packing, placement and routing. It maps technology mapped netlist to hypothetical FPGA specified by user. A low level bitstream manipulation tools BitMAT [7] and "BITMAN" [8] targeted Virtex- II FPGAs. A Java library named "abit” for direct manipulation of Atmel FPSLIC series bitstream and partial reconfiguration found in [9]. In [10] an open source tool based on library of micro-bitstream created for primitives and merged for creating larger design with simple merging operations. An open source tool set for reconfigurable computing presented in [11] is based on C++. Torc infrastructure can read, write, and manipulate EDIF and XDL netlist as well as Xilinx bitstream packets (but not configuration frame internals). Currently supported devices include all Virtex, VirtexE, Virtex2 pro, Virtex5, Virtex6, Virtex6L devices. PARBIT a tool in [12] extracts and relocates Virtex partial bitstream. The RapidSmith project [13] provide exclusive platform for implementing experimental idea, algorithms on Xilinx FPGAs.

Knowing bitstream format opens innovative approach for development in FPGA technology. Mostly, such type of work depends upon available documentation from vendor. But the vendor does open fair amount of information for end users and keeps certain information closed to avoid cloning of their devices. In this paper we have proposed a simple approach to map configuration bitstream to FPGA resources.

\section{FPGA GENERIC ARCHITECTURE AND DESIGN FLOW}

FPGA means field programmable gate array. Any arbitrary digital function is implemented in FPGA. It can be programmed to implement desired hardware on it. Basic building blocks of FPGAs are configuration logic blocks (CLBs), I/O blocks, switch blocks, routing resources, memory blocks, and other dedicated functional block. The Fig. 2 gives general architecture of FPGA and Spartan -3A FPGA layout as viewed in PlanAhead design tool.

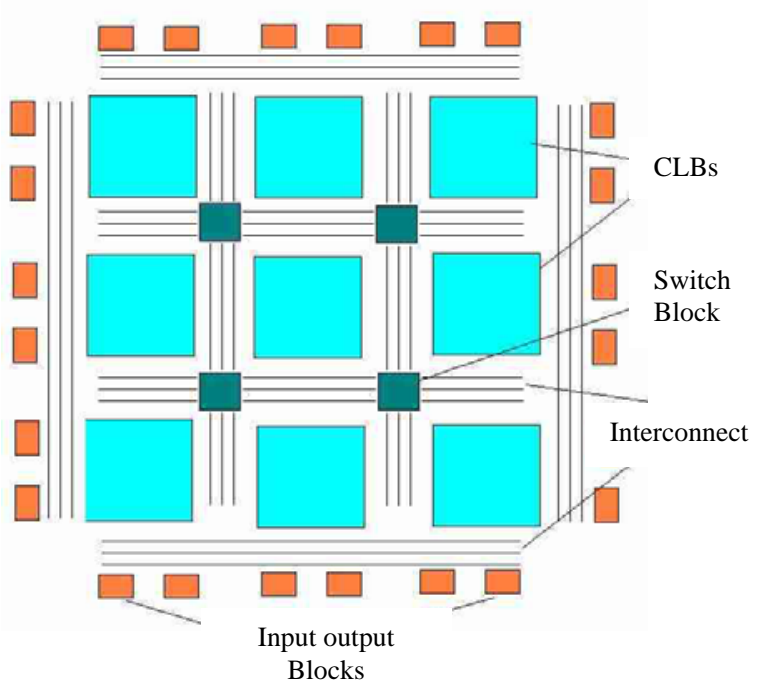

Fig. 2 (a) General architecture of FPGA

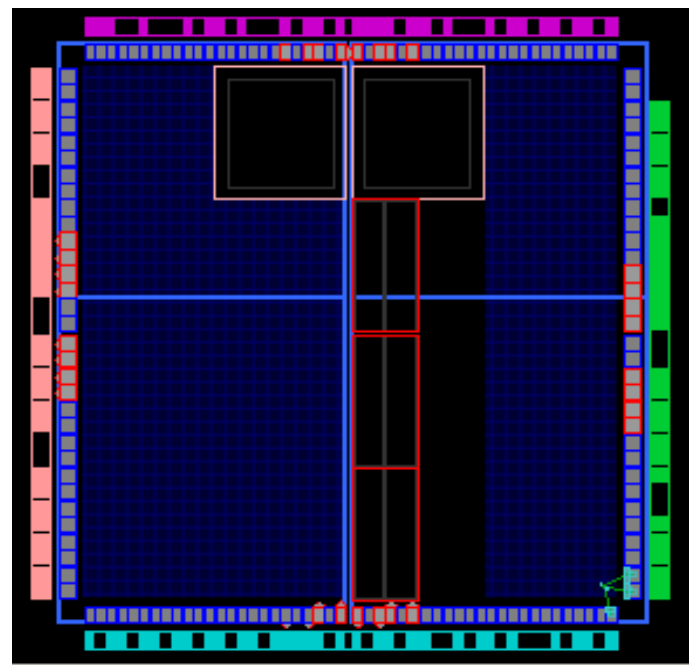

Fig. 2 (b) Spartan-3A layout in Plan Ahead

Fig. 2 FPGA architecture and PlanAhead view of Spartan-3A layout

A typical FPGA design flow consists of different stages like design entry, synthesis, mapping, place and route and bitstream generation. Fig. 3 depicts FPGA design flow. Three main stages of design are design entry and synthesis, design implementation and design verification [14]. Design entry is made either in schematic capture or in hardware design languages or with both. Synthesis is the process by which a given design is converted to logical design format (EDIF) or NGC file (by using Xilinx Synthesis Technology GUI). Design implementation converts the logical design format into Native Circuit Description (NCD) file. NCD file contains the physical 
information of FPGAs. The bitstream file generated after this stage is used to program the FPGA. In the last stage designer verifies whether the circuit meets timing and functionality requirements

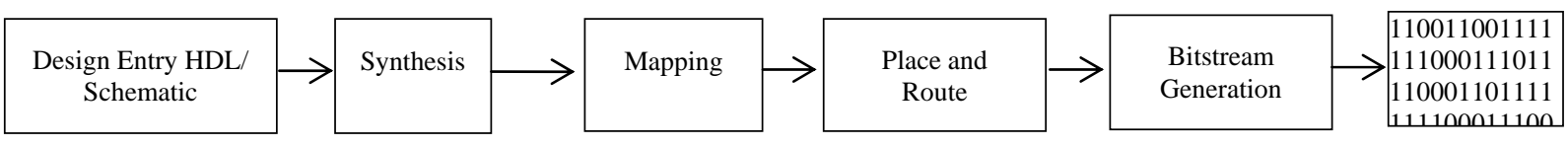

Fig. 3 Typical FPGA design flow

\section{BACKGROUND: HDL-NCD-XDL}

Listing 1 shows VHDL codes for two input AND gate. DPI1 and DIP2 are the two input ports and LED1 is the output port. Fig. 4 represents RTL schematic and Fig. 5 shows schematic of AND gate generated after VHDL codes compiled on Xilinx ISE Design Suite 14.7. Device utilization summary is given in Table 1. Other relevant information is generated by the tool. Here we gathered information necessary for mapping of FPGA configuration bitstream to its resources. "andGate.ncd" file is located in project folder directory. It is Native Circuit Description file which contains how HDL codes are mapped to physical resources like LUTs, flip flops, I/Os, routing etc. on FPGA. But it is not in human readable format. Xilinx Design Language is used to convert NCD file into human readable format. A command like "xdl -ncd2xdl andGate.ncd" converts NCD to XDL file. and Gate.xdc contains human readable information. A brief introduction of XDL is given by illustrating "andGate" example.

Listing 1. VHDL code of AND gate
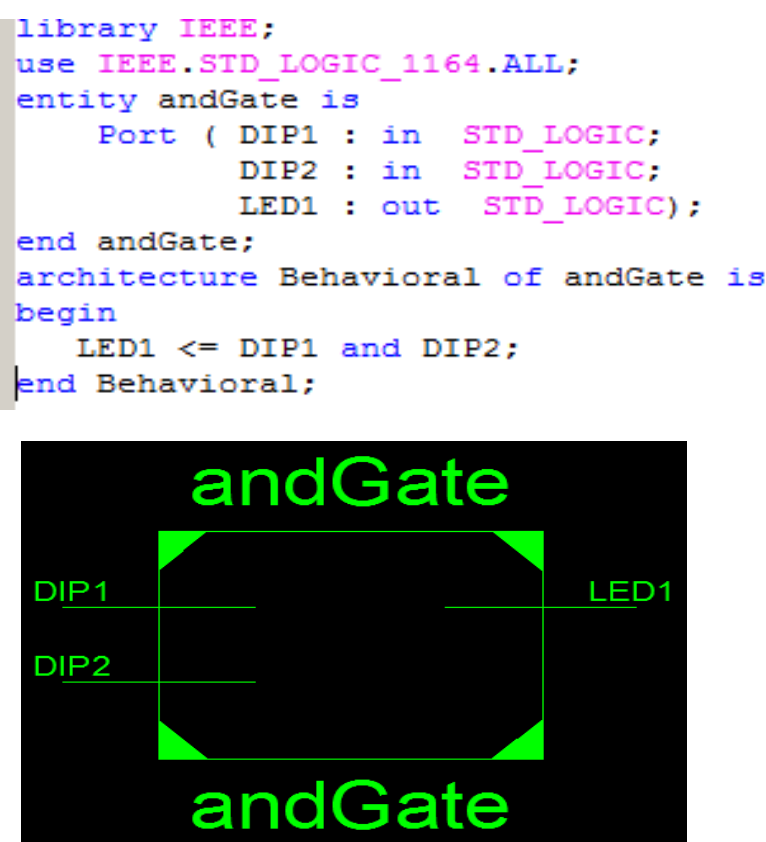

Fig.4 RTL schematic

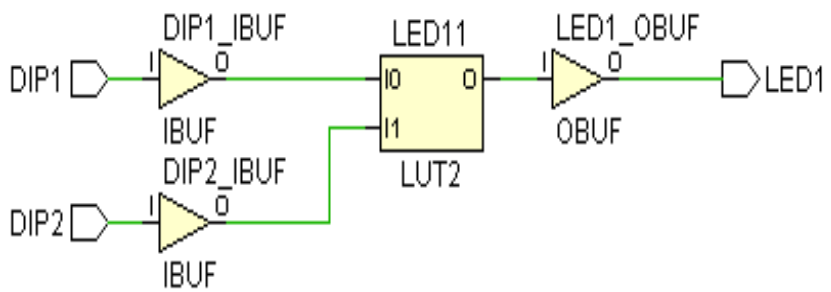

Fig. 5 Schematic of AND gate 
Table 1. Device Utilization Summary

\begin{tabular}{|l|c|c|c|}
\hline Logic Utilization & used & Available & Utilization \\
\hline Number of 4 input LUTs & 1 & 1,408 & $1 \%$ \\
\hline Number of occupied Slices & 1 & 704 & $1 \%$ \\
\hline Number of Slices containing only related logic & 1 & 1 & $100 \%$ \\
\hline Number of Slices containing unrelated logic & 0 & 1 & $0 \%$ \\
\hline Total Number of 4 input LUTs & 1 & 1,408 & $1 \%$ \\
\hline Number of bonded IOBs & 3 & 108 & $2 \%$ \\
\hline Average Fanout of Non-Clock Nets & 1.00 & & \\
\hline
\end{tabular}

\section{Xilinx Design Languages (XDL)}

The NCD file contains all necessary information to implement the design on FPGA. But the NCD file format is Xilinx specific and it is not possible to analyze it. However, Xilinx has provided Xilinx Design Language in Xilinx ISE Design suite. It converts NCD file into XDL file which is human readable. Less information is available on XDL documentation.

\section{XDL File Format}

An XDL file is a text file contains information about the name of the design, intended Xilinx FPGA device and cfg attribute which allows user to configure the certain aspects of the design. It also lists logical slices, nets for routing design. The keyword "inst" is used to begin the instance statement. Instance is the instance of FPGA primitive. After the instance keyword the name of the instance is mentioned. It is followed by instance type. Placement of the slice is described by the keyword "placed" or "unplaced" after the instance type. A string beginning with "cfg” keyword used to configure the LUT contents and other functionality. Instance statement for DIP1 in case of AND gate example is given below.

inst "DIP1" "IBUF", placed RIOIS_X17Y1 P78

cfg " DELAY_ADJ_ATTRBOX: :FIXED GTSATTRBOX: :\#OFF IBUF_DELAY_VALUE: :DLY@

ICEINV::\#OFF ICLK1INV: :\#OFF ICLK2INV::\#OFF IDDRIN_MUX::\#OFF IFD_DELAY_VALUE: :DLYO

IFF1::\#OFF IFF1_INIT_ATTR::\#OFF IFF1_SR_ATTR::\#OFF IFF2::\#OFF IFF2_INIT_ATTR::\#OFF

IFF2_SR_ATTR::\#OFF IFFATTRBOX::\#OFF IFFDMUX::\#OFF IMUX::1 IOATTRBOX::LVCMOS25

IREV_USED: :\#OFF ISR_USED: :\#OFF MISRATTRBOX: :\#OFF MISR_CLK_SELECT: :\#OFF

O1INV::\#OFF 01_DDRMUX: :\#OFF 02INV: :\#OFF 02_DDRMUX: :\#OFF OCEINV: :\#OFF

ODDROUT1_MUX::\#OFF ODDROUT2_MUX::\#OFF OFF1::\#OFF OFF1_INIT_ATTR: :\#OFF

OFF1_SR_ATTR: :\#OFF OFF2: :\#OFF OFF2_INIT_ATTR: :\#OFFOFF2_SR_ATTR: :\#OFF

OFFATTRBOX: :\#OFF OMUX: :\#OFF OREV_USED: :\#OFF OSR_USED: :\#OFF OTCLK1INV: :\#OFF

OTCLK2INV: :\#OFF PCICE_MUX: :\#OFF PCIRDY_MUX: :\#OFF PULL::\#OFF REVINV: :\#OFF

SEL_MUX: :๑ SLEW: :\#OFF SRINV: :\#OFF T1INV: :\#OFF T2INV: :\#OFF TCEINV: :\#OFF

TFF1: :\#OFF TFF1_INIT_ATTR: :\#OFF TFF1_SR_ATTR: :\#OFF TFF2::\#OFF TFF2_INIT_ATTR: :\#OFF

TFF2_SR_ATTR: :\#OFF TFFATTRBOX: :\#OFF TMUX: :\#OFF TREV_USED: :\#OFF TSMUX: :\#OFF

TSR_USED: :\#OFF T_USED: :\#OFF DELAY_ADJ_BBOX:DIP1.DELAY_ADJ : INBUF:DIP1_IBUF :

PAD:DIP1: "

;

The logic blocks are interconnected by the nets. FPGA fabric contains the static wires. The nets are used for routing purpose. A net has "outpin” as one starting point and "inpin” as endpoints (it can be multiple). The configuration in FPGAs routing structure is the connection between these nets. In Xilinx terminology these are called pips (programmable interconnect points). The inpin and outpin are connected by pip declaration. A set of pips declarations constitutes a single net. Pip is declared as "pip LOC S -> E" where pip is keyword, LOC is the location of pip, $\mathrm{S}$ is the starting point and $\mathrm{E}$ is the endpoint of the wire. Finally summary statements give statics of modules, instances and nets used in the design. Listing 2 summaries the description of XDL file format for "andGate" example compiled for XC3S50A Spartan-3A FPGA. Latter in the paper this design is named as "default" as it is compiled without any constraint. 
Listing 2. XDL file from NCD file for AND gate default design example.

\# XDL NCD CONVERSION MODE \$Revision: 1.01\$

\# time: Sat Jan 27 00:55:22 2018

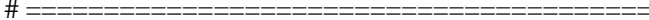

\# The syntax for the design statement is:

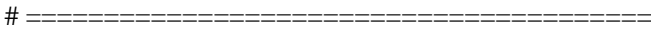

design "andGate" xc3s50atq144-5 v3.2 ,

cfg "

DESIGN_PROP::PK_NGMTIMESTAMP:1516987046"

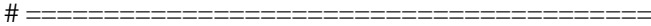

inst "DIP1" "IBUF",placed RIOIS_X17Y1 P78

inst "DIP2" "IBUF",placed BIOIS_X16Y0 P72

inst "LED1" "IOB",placed RIOIS_X17Y1 P76

inst "LED1_OBUF" "SLICEL",placed CLB_X16Y1

SLICE_X23Y0

$\#============+$
net "DIP1_IBUF" ,

outpin "DIP1" I,

inpin "LED1_OBUF" G1 ,

pip CLB_X16Y1 G1_B1 -> G1_B_PINWIRE1,

pip CLB_X16Y1 OMUX_W1 -> G1_B1 ,

pip RIOIS_X17Y1 I1_PINWIRE -> IOIS_Y1,

pip RIOIS_X17Y1 IOIS_Y1 -> OMUX1,

$$
\text { ; }
$$

Note: In the above listing "cfg" keyword and its listing is not included.

\section{METHODOLOGY AND IMPLEMENTATION}

Initially an AND gate was designed and implemented in VHDL hardware description language without any constraints. Latter same VHDL codes were used to implement AND gate design with different constraints so that AND gate will map to different location. The pblock is used for restricting AND gate on FPGA layout along with defined I/O pins location is mentioned in the Table 2. In each case, design is compiled on Xilinx ISE 14.7 Design Suite [15]. Ensure that generate debug is enabled in process properties dialog box. This is shown in Fig. 6. This will generate FAR address for each configuration frame.

Fig. 7 shows location of CLBs on FPGA layout selected for experimentation for default, const_1 and const_2. After compiling same AND gate design with different constraints of slices and I/O pins assignment, a bit file database is prepared. Each "andGate.bit" file is analysed separately. Configuration bitstream file is opened in Hex Editor Neo. A frame containing all zero in bitstream file is selected. After that with the help of hex editor all such frames containing zeros are found out. An observation of starting portion and ending portion of the frame is noted. The important part of bitstream is the configuration data and its addresses. For all the designs, FAR addresses containing configuration information are tabulated. Frame addresses which points to zero configuration information are neglected. This process will separate out bitstream information into four parts: starting, ending, design information and no information of design. The design information portion is pointed by FAR register addresses. PlanAhead tool is used to observe device view for mapped resources on Spartan-3A in FPGA layout. An FPGA editor [16] is used to view how resources are placed and routed.

Table 2. Floor planning of AND gate and constraints

\begin{tabular}{|c|c|c|c|c|c|}
\hline Const. No. & Pblock Range & CLB & DIP1 & DIP2 & LED1 \\
\hline \multirow{2}{*}{ Default } & \multirow{2}{*}{ NA } & \multirow{2}{*}{ CLB_X16Y1 } & RIOSIS_X17Y1 & BIOIS_X16Y0 & RIOIS_X17Y1 \\
\hline & & & P78 & P72 & P76 \\
\hline \multirow{2}{*}{ Const_1 } & \multirow{2}{*}{ SLICE_X20Y28:SLICE_X21Y29 } & \multirow{2}{*}{ CLB_X15Y15 } & RIOSIS_X17Y15 & RIOSIS_X17Y15 & RIOSIS_X17Y14 \\
\hline & & & P101 & P103 & P104 \\
\hline \multirow{2}{*}{ Const_2 } & \multirow{2}{*}{ SLICE_X0Y30:SLICE_X1Y31 } & \multirow{2}{*}{ CLB_X1Y16 } & TOIOIB_X1Y17 & TOIOIB_X1Y17 & TOIOIB_X1Y17 \\
\hline & & & P141 & P140 & P143 \\
\hline \multirow{2}{*}{ Const_3 } & \multirow{2}{*}{ SLICE_X0Y0:SLICE_X1Y1 } & \multirow{2}{*}{ CLB_X1Y1 } & BIOIB_X1Y0 & BIOIB_X1Y0 & BIOIS_X2Y0 P39 \\
\hline & & & P37 & P38 & P39 \\
\hline \multirow{2}{*}{ Const_4 } & \multirow{2}{*}{ SLICE_X10Y20:SLICE_X11Y21 } & \multirow{2}{*}{ CLB_X6Y11 } & LIOIS_PCIX0Y11 & LIOIS_PCIX0Y11 & LIOIS_PCIX0Y11 \\
\hline & & & $\mathrm{P} 12$ & $\mathrm{P} 13$ & $\mathrm{P} 10$ \\
\hline \multirow{2}{*}{ Const_5 } & \multirow{2}{*}{ SLICE_X2Y20:SLICE_X3Y21 } & \multirow{2}{*}{ CLB_X2Y11 } & LIOIS_PCIX0Y11 & LIOIS_PCIX0Y11 & LIOIS_PCIX0Y11 \\
\hline & & & $\mathrm{P} 12$ & $\mathrm{P} 13$ & $\mathrm{P} 10$ \\
\hline \multirow{2}{*}{ Const_6 } & \multirow{2}{*}{ SLICE_X4Y20:SLICE_X5Y21 } & \multirow{2}{*}{ CLB_X3Y11 } & LIOIS_PCIX0Y11 & LIOIS_PCIX0Y11 & LIOIS_PCIX0Y11 \\
\hline & & & $\mathrm{P} 12$ & $\mathrm{P} 13$ & P10 \\
\hline \multirow{2}{*}{ Const_7 } & \multirow{2}{*}{ SLICE_X6Y20:SLICE_X7Y21 } & \multirow{2}{*}{ CLB_X4Y11 } & LIOIS_PCIX0Y11 & LIOIS_PCIX0Y11 & LIOIS_PCIX0Y11 \\
\hline & & & $\mathrm{P} 12$ & $\mathrm{P} 13$ & $\mathrm{P} 10$ \\
\hline
\end{tabular}




\begin{tabular}{|c|c|c|c|c|c|}
\hline Const. No. & Pblock Range & CLB & DIP1 & DIP2 & LED1 \\
\hline \multirow{2}{*}{ Const_8 } & \multirow{2}{*}{ SLICE_X8Y20:SLICE_X9Y21 } & \multirow{2}{*}{ CLB_X5Y11 } & LIOIS_PCIX0Y11 & LIOIS_PCIX0Y11 & LIOIS_PCIX0Y11 \\
\hline & & & $\mathrm{P} 12$ & P13 & P10 \\
\hline \multirow{2}{*}{ Const_9 } & \multirow{2}{*}{ SLICE_X2Y22:SLICE_X3Y23 } & \multirow{2}{*}{ CLB_X2Y12 } & LIOIS_PCIX0Y11 & LIOIS_PCIX0Y11 & LIOIS_PCIX0Y11 \\
\hline & & & P12 & $\mathrm{P} 13$ & P10 \\
\hline \multirow{2}{*}{ Const_10 } & \multirow{2}{*}{ SLICE_X2Y26:SLICE_X3Y27 } & \multirow{2}{*}{ CLB_X2Y14 } & LIOIS_PCIX0Y11 & LIOIS_PCIX0Y11 & LIOIS_PCIX0Y11 \\
\hline & & & P12 & P13 & $\mathrm{P} 10$ \\
\hline
\end{tabular}

\begin{tabular}{|c|c|c|c|}
\hline Category & Switch Name & Property Name & \\
\hline \multirow{10}{*}{$\begin{array}{l}\text {.. General Options } \\
\text { - Configuration Options } \\
\text { - Startup Options } \\
\text { - Readback Options } \\
\text {.. Suspend/Wake Options }\end{array}$} & $-d$ & Run Design Rules Checker (DRC) & $\sqrt{\square}$ \\
\hline & $-\mathrm{j}$ & Create Bit File & $\sqrt{\checkmark}$ \\
\hline & -g Binary: & Create Binary Configuration File & $\sqrt{\square}$ \\
\hline & $-b$ & Create ASCII Configuration File & $\sqrt{\checkmark}$ \\
\hline & -g IEEE1532: & Create IEEE 1532 Configuration File & $\Gamma$ \\
\hline & -g Compress & Enable BitStream Compression & $\Gamma$ \\
\hline & $-g$ DebugBitstream: & Enable Debugging of Serial Mode BitStream & $\sqrt{\checkmark}$ \\
\hline & -g CRC: & Enable Cyclic Redundancy Checking (CRC) & $\sqrt{\checkmark}$ \\
\hline & -g reset_on_error: & Retry Configuration if CRC Error Occurs & $\Gamma$ \\
\hline & & Other Bitgen Command Line Options & \\
\hline
\end{tabular}

Fig. 6 Enable debugging of bitstream in process properties of generate Programming

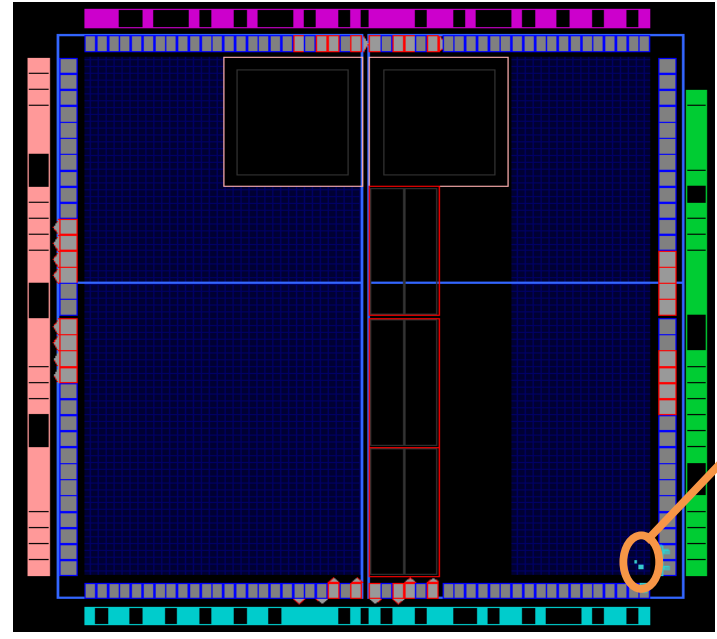

A. Default on device layout

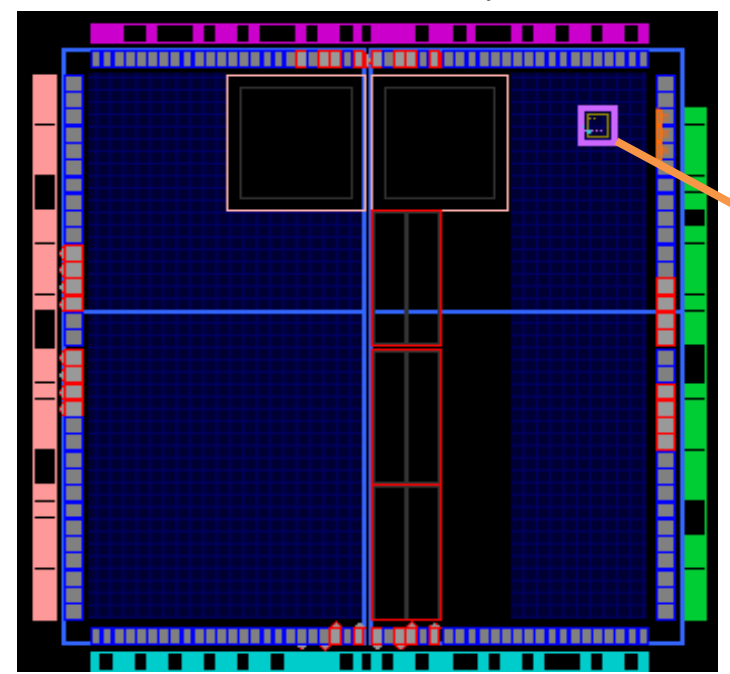

B. Const_1 on device layout
A. Default zoom view
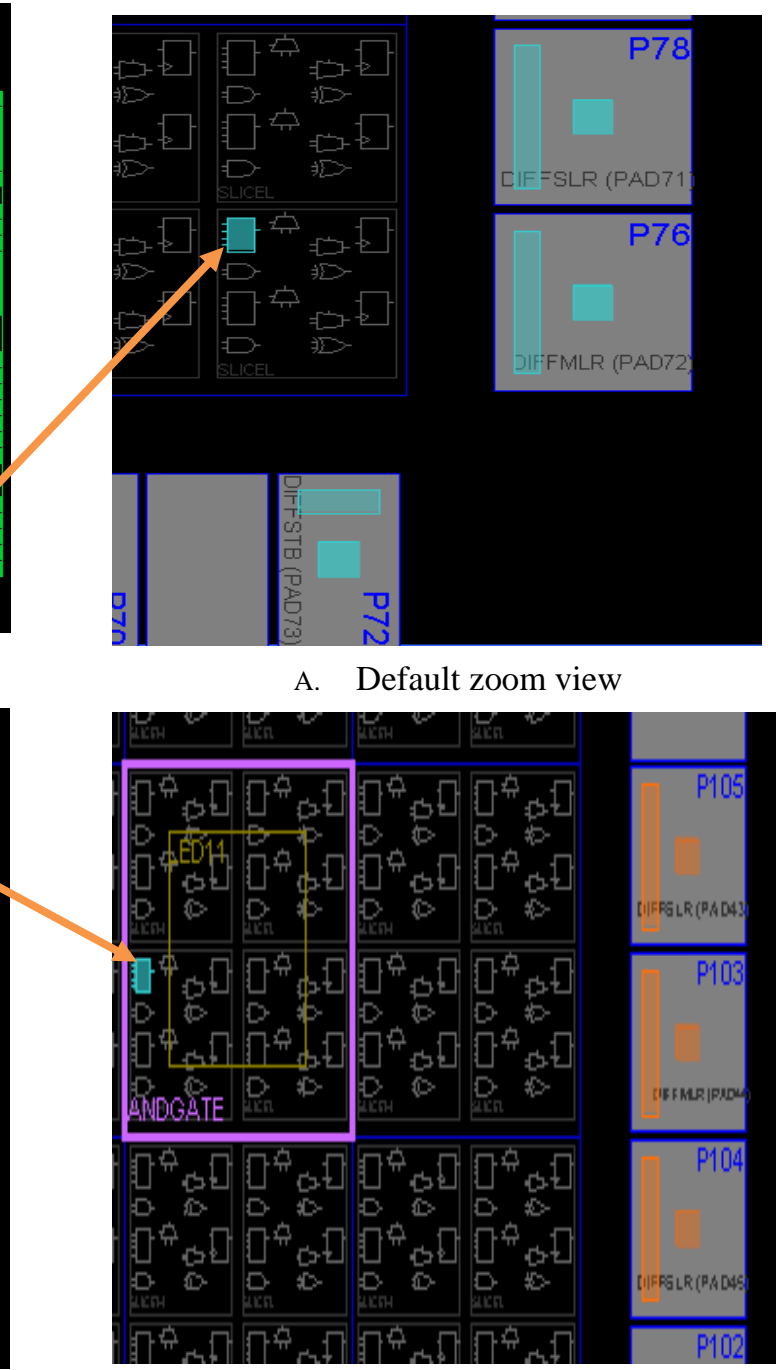

B. Const_1 zoom view 


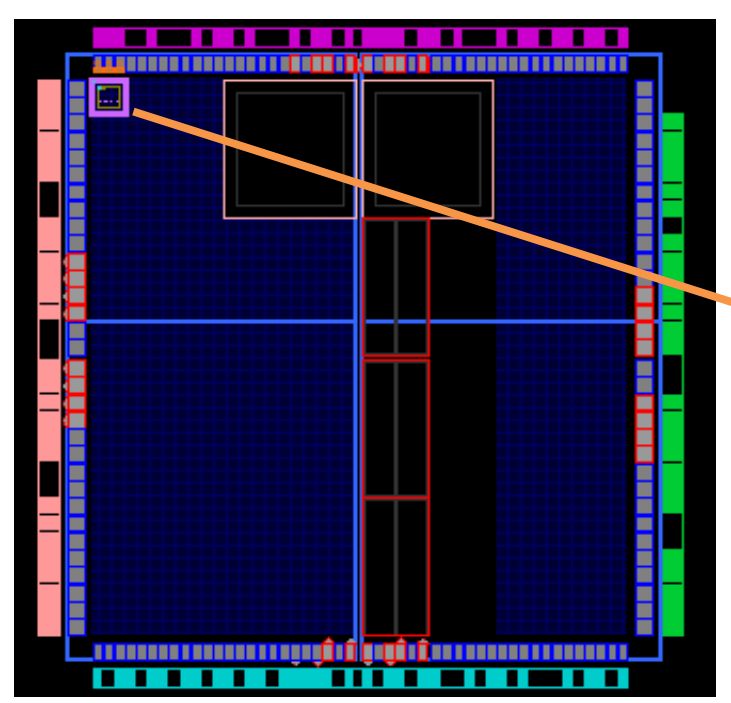

C. Const_2 on device layout

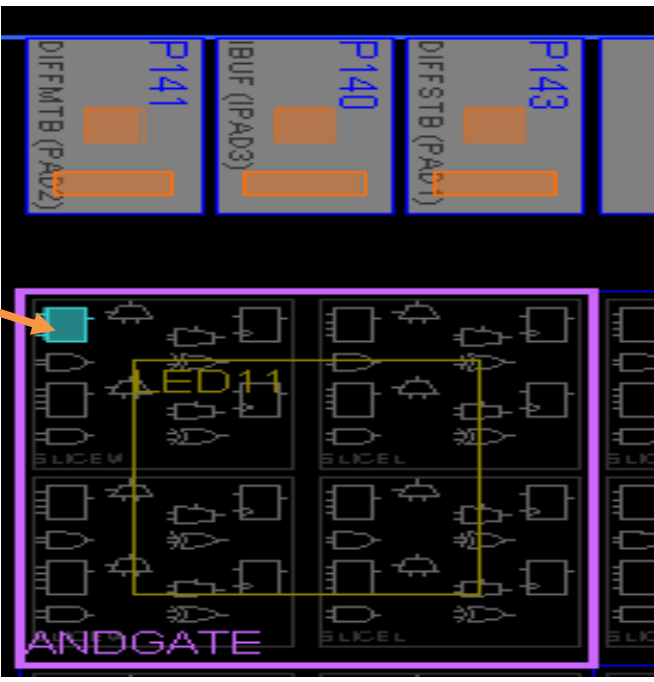

C. Const_2 zoom view

Fig. 7 Different CLB location selections with constraints

\section{RESULTS AND DISCUSSION}

\section{Analysis of Configuration Bitstream}

Bitstream files consist of a start header (Fig. 8), configuration data, bitstream final command (Fig.10) and start-up sequence. The start header contains information like design name, target device, file modification time, dummy word, synchronization word, reset CRC, device ID code, etc. The packets contain configuration commands and configuration data. The small state machine in FPGA controls configuration of programmable resources on FPGA. The command register in FPGA is either read or written by configuration information in data frames in bitstream. Always action taken by state machines is as per the command registers data. The data frames are loaded with configuration data as pointed by the frame address register (FAR). Configuration data frames can be, single frame, set of frames or full configuration space. During continuous writing of configuration data into FPGA, the FAR is automatically incremented after every FDRI write [17]. In this case frame length register (FLR) contains the length of data frame. It is written before the initiation of command to load block of data. Finally START, CRC checksum DESYNCH command are executed towards the end of bitstream file as shown in Figure 10 and Figure 11. Figure 8 and Fig. 10 shows starting and ending part of the bitstream file of AND gate example. The interpretation of each word in the frame is explained in Fig. 9 and Fig.11.

\begin{tabular}{|c|c|c|c|c|c|c|c|c|c|c|c|c|c|c|c|c|}
\hline & & 01 & 02 & 03 & 04 & 05 & 06 & 07 & 08 & 09 & $0 \mathrm{a}$ & $\mathrm{Ob}$ & $0 \mathrm{c}$ & od & De & of \\
\hline & & & & & & & & & & & & & & & & \\
\hline & & & & & & & & & & & & & & & & \\
\hline & 0221 & & & & & & & & & & & & & & & 10000 \\
\hline 00000 & 3261 & 0000 & 3281 & 0000 & 3341 & & 3362 & 0000 & 0000 & & 0000 & & & & & 0000 \\
\hline & $004 a$ & & & & & & & & & & & & & & & 0000 \\
\hline & 0000 & 0000 & 0000 & 0000 & 0000 & & 0000 & 0000 & 0000 & & 0000 & & 0000 & & & 0000 \\
\hline 0000000 & 0000 & 0000 & 0000 & 0000 & 0000 & 0000 & 0000 & 0000 & 0000 & 0000 & 0000 & 0000 & 0000 & 0000 & 0000 & 0000 \\
\hline
\end{tabular}




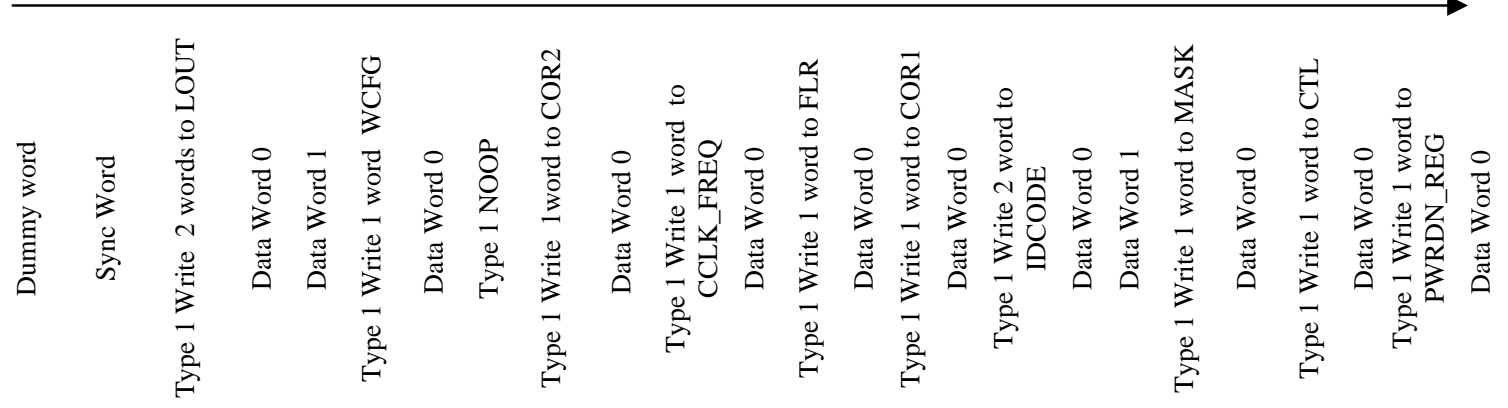

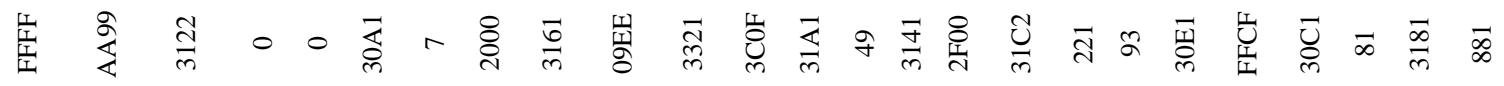

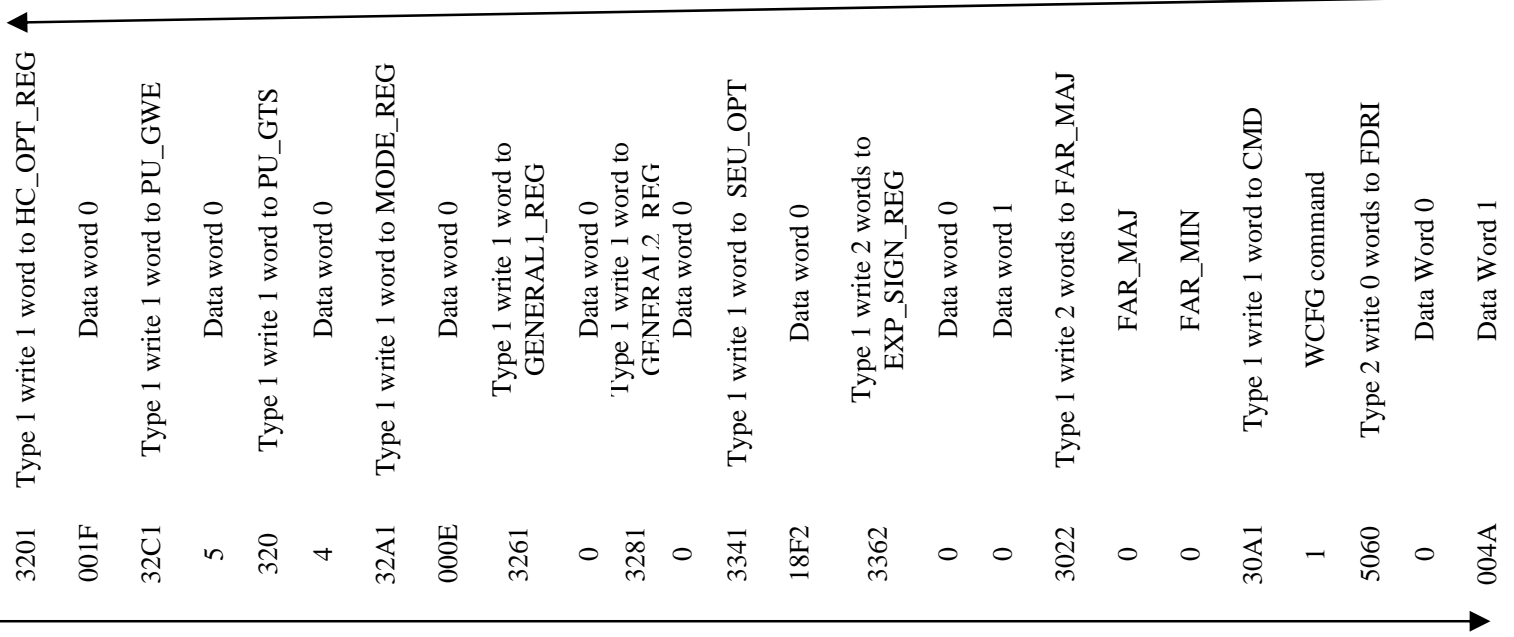

Fig.9 Decoding of start portion of bitstream file

\begin{tabular}{|c|c|c|c|c|c|c|c|c|}
\hline $0000 \mathrm{e} 620$ & 00000000 & 00000000 & 00000000 & 00000000 & 00000000 & 00000000 & 00000000 & 00000000 \\
\hline $0000 \mathrm{e} 640$ & 00000000 & 00000000 & 00000000 & 00000000 & 00000000 & 00000000 & 00000000 & 00000000 \\
\hline $0000 \mathrm{e} 660$ & 00000000 & 00000000 & 00000000 & 00000000 & 00000000 & 00003002 & 001377 e7 & $30 a 1000 a$ \\
\hline 0000 e 680 & $30 a 10003$ & 20002000 & 20002000 & $30 \mathrm{a} 10005$ & $30 \mathrm{e} 10081$ & $30 \mathrm{c} 10081$ & $3002000 \mathrm{e}$ & $79 \mathrm{c} 730 \mathrm{a} 1$ \\
\hline $0000 \mathrm{e} 6 \mathrm{a} 0$ & $000 \mathrm{~d} 2000$ & 20002000 & 20002000 & 20002000 & 20002000 & 20002000 & 20002000 & 20002000 \\
\hline & 2000 & & & & & & & \\
\hline
\end{tabular}

Fig.10 End portion of bitstream file

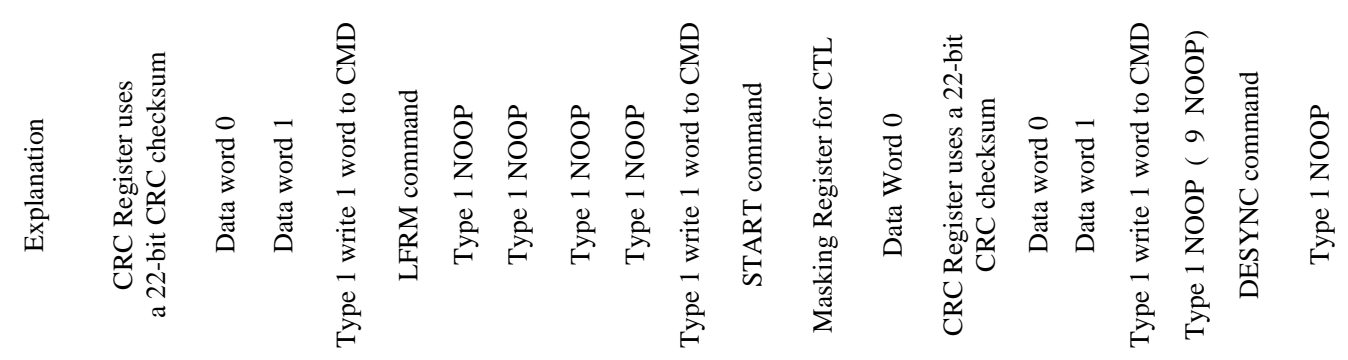

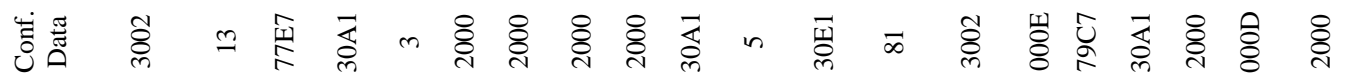

Fig. 11 Decoding of end portion of bitstream file 


\section{Decoding of Frame Address Register (FAR)}

Frame addresses decoded for each of the design is shown in Table 3. All designs belongs to block type 0 containing CLBs, IOBs information. Major address selects major column and minor address selects memorycell address line within a major column. Column labelled with " $M$ " indicates major address whereas " $m$ " indicates minor address of frames. The addressing scheme gives the idea of the columns where the design lies vertically. Frame addresses and total number of frames required for implementation of design varies according to design type.

Table 3. Decoding of frame address register

\begin{tabular}{|c|c|c|c|c|c|c|c|c|c|c|c|c|c|c|c|c|c|c|c|c|c|c|}
\hline & \multicolumn{2}{|c|}{ default } & \multicolumn{2}{|c|}{ Const_1 } & \multicolumn{2}{|c|}{ Const_2 } & \multicolumn{2}{|c|}{ Const_3 } & \multicolumn{2}{|c|}{ Const_4 } & \multicolumn{2}{|c|}{ Const_5 } & \multicolumn{2}{|c|}{ Const_6 } & \multicolumn{2}{|c|}{ Const_7 } & \multicolumn{2}{|c|}{ Const_8 } & \multicolumn{2}{|c|}{ Const_9 } & \multicolumn{2}{|c|}{ Const_10 } \\
\hline $\begin{array}{l}\mathrm{S} \\
\mathrm{N}\end{array}$ & $\mathrm{M}$ & $\mathrm{m}$ & M & $\mathrm{m}$ & M & $\mathrm{m}$ & M & $\mathrm{m}$ & M & $\mathrm{m}$ & M & M & M & $\mathrm{m}$ & M & $\mathrm{m}$ & M & $\mathrm{m}$ & M & $\mathrm{m}$ & M & $\mathrm{m}$ \\
\hline 1 & 1 & 0 & 1 & 0 & 1 & 0 & 1 & 0 & 1 & 0 & 1 & 0 & 1 & 0 & 1 & 0 & 1 & 0 & 1 & 0 & 1 & 0 \\
\hline 2 & 1 & 1 & 1 & 1 & 1 & 1 & 1 & 1 & 1 & 1 & 1 & 1 & 1 & 1 & 1 & 1 & 1 & 1 & 1 & 1 & 1 & 1 \\
\hline 3 & 14 & 3 & 13 & 0 & 3 & 0 & 3 & 3 & 2 & 0 & 2 & 0 & 2 & 0 & 2 & 0 & 2 & 0 & 2 & 0 & 2 & 0 \\
\hline 4 & 14 & 4 & 13 & 1 & 3 & 1 & 3 & 5 & 2 & 1 & 2 & 1 & 2 & 1 & 2 & 1 & 2 & 1 & 2 & 1 & 2 & 1 \\
\hline 5 & 14 & 5 & 13 & 7 & 3 & 4 & 3 & 6 & 2 & 10 & 2 & 9 & 2 & 10 & 2 & 9 & 2 & 9 & 2 & 10 & 2 & 6 \\
\hline 6 & 14 & 6 & 13 & 9 & 3 & 5 & 3 & 7 & 2 & 14 & 2 & 13 & 2 & 13 & 2 & 11 & 2 & 17 & 2 & 14 & 2 & 7 \\
\hline 7 & 14 & 7 & 13 & 11 & 3 & 6 & 3 & 8 & 2 & 15 & 2 & 15 & 2 & 14 & 2 & 12 & 2 & 18 & 2 & 15 & 2 & 9 \\
\hline 8 & 14 & 8 & 14 & 14 & 3 & 7 & 3 & 9 & 2 & 17 & 3 & 13 & 2 & 15 & 3 & 13 & 4 & 13 & 2 & 17 & 3 & 13 \\
\hline 9 & 14 & 10 & 14 & 15 & 3 & 8 & 3 & 10 & 2 & 18 & 3 & 14 & 4 & 13 & 3 & 14 & 4 & 14 & 2 & 18 & 4 & 0 \\
\hline 10 & 14 & 11 & 15 & 0 & 3 & 9 & 3 & 11 & 8 & 0 & 4 & 0 & 4 & 15 & 5 & 13 & 6 & 13 & 8 & 0 & 4 & 1 \\
\hline 11 & 14 & 12 & 15 & 1 & 3 & 10 & 3 & 12 & 8 & 1 & 4 & 1 & 5 & 0 & 5 & 14 & 6 & 14 & 8 & 1 & 4 & 6 \\
\hline 12 & 14 & 13 & 15 & 9 & 3 & 11 & 3 & 13 & 8 & 8 & 4 & 6 & 5 & 1 & 6 & 0 & 7 & 0 & 8 & 8 & 4 & 8 \\
\hline 13 & 15 & 0 & 15 & 14 & 3 & 12 & 3 & 14 & 8 & 9 & 4 & 8 & 5 & 8 & 6 & 1 & 7 & 1 & 8 & 9 & 4 & 9 \\
\hline 14 & 15 & 1 & 16 & 0 & 3 & 13 & 4 & 0 & 8 & 11 & 4 & 9 & 5 & 9 & 6 & 6 & 7 & 6 & 8 & 11 & 4 & 11 \\
\hline 15 & 15 & 7 & 16 & 1 & 3 & 15 & 4 & 1 & 8 & 12 & 4 & 11 & 5 & 11 & 6 & 9 & 7 & 9 & 8 & 12 & 4 & 12 \\
\hline 16 & 15 & 11 & & & 3 & 16 & 4 & 8 & 8 & 13 & 4 & 12 & 5 & 12 & 6 & 12 & 7 & 10 & 8 & 13 & & \\
\hline 17 & 15 & 12 & & & 3 & 17 & 4 & 10 & 8 & 17 & & & 5 & 17 & & & 7 & 11 & 8 & 17 & & \\
\hline 18 & 16 & 0 & & & 3 & 18 & 4 & 11 & & & & & & & & & 7 & 12 & & & & \\
\hline 19 & 16 & 1 & & & 4 & 18 & 4 & 12 & & & & & & & & & 8 & 14 & & & & \\
\hline 20 & & & & & & & 4 & 14 & & & & & & & & & & & & & & \\
\hline 21 & & & & & & & 4 & 15 & & & & & & & & & & & & & & \\
\hline
\end{tabular}

Note: " $M$ " indicates Major Address and " $m$ " indicates minor address.

\section{Matching and Mismatching Patterns in Configuration Bitstream}

Extended Spartan-3A device, XC3S50A has 367 device frames. Result of match and mismatch pattern in configuration bitstream shown in Fig. 12.

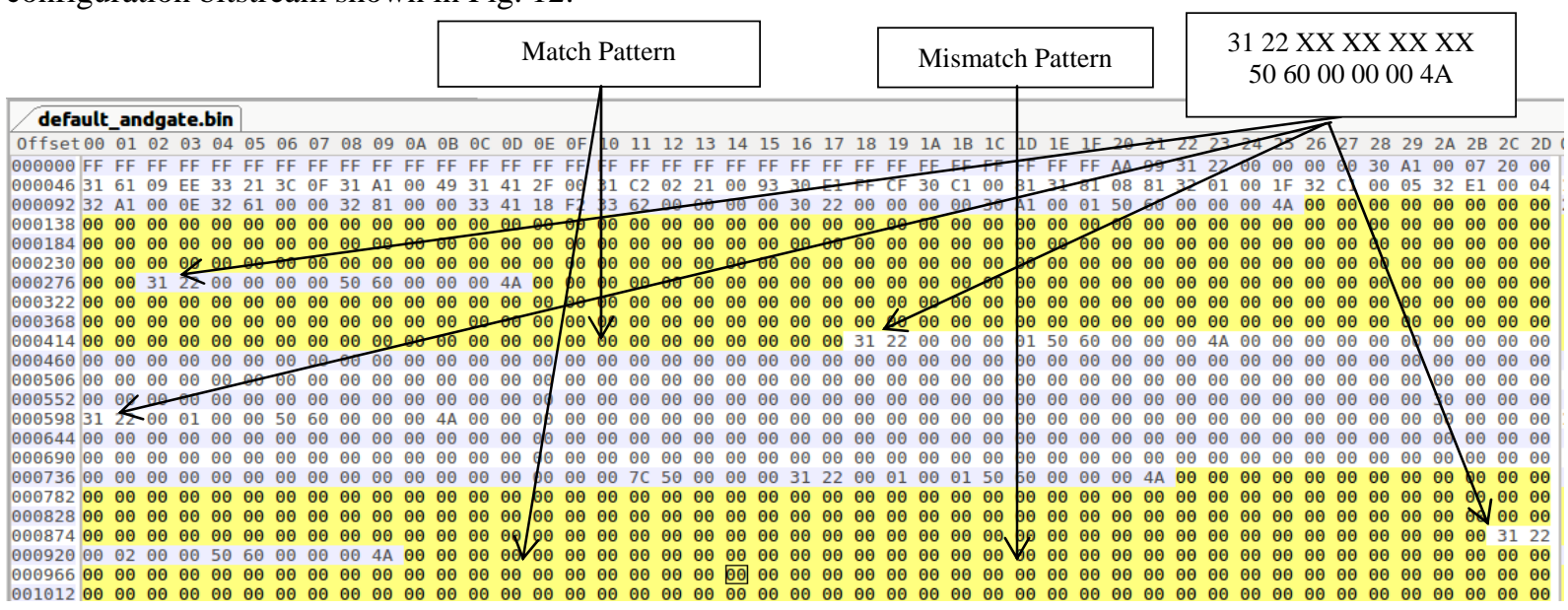

Fig.12 Match and mismatch patterns in configuration bitstream 
Table 4 indicated the count of match and mismatch patterns as observed in each bitstream file. Also, it gives total count of combined pattern. The frame address for which mismatch pattern were observed are mentioned in Table 3. The mismatch pattern in bitstream file contains information about the implemented designs.

Table 4. Match and Mismatch Patterns in Configuration Bitstream

\begin{tabular}{|c|c|c|c|}
\hline Constraint No & Pattern Match Found & Pattern Mismatch & Total Pattern \\
\hline Default & 349 & 19 & 368 \\
\hline Const_1 & 353 & 15 & 368 \\
\hline Const_2 & 349 & 19 & 368 \\
\hline Const_3 & 347 & 21 & 368 \\
\hline Const_4 & 351 & 17 & 368 \\
\hline Const_5 & 352 & 16 & 368 \\
\hline Const_6 & 351 & 17 & 368 \\
\hline Const_7 & 351 & 16 & 368 \\
\hline Const_8 & 349 & 19 & 368 \\
\hline Const_9 & 353 & 15 & 368 \\
\hline Const_10 & 353 & 15 & 368 \\
\hline
\end{tabular}

Though the HDL codes are same for each design, the location of each design is different on FPGA tiles. For each of the design the mismatch patterns count is different. This is because the routing structure is different for each design. Routing of designs viewed in FPGA editor. Fig. 13 (a) depicts routing structure for default design. Three switch boxes are used for routing. Default design is located by placement and routing tool at bottom right corner of the layout. Fig. 13(b) depicts routing structure for design with const_4. This design resides in middle portion of the layout.

For the default design LUT belongs to SLICE_X23Y0 of CLB_X16Y1. This is also included in statement, “inst "LED1_OBUF" "SLICEL", placed CLB_X16Y1 SLICE_X23Y0” in XDL file generated from NCD file. Also the instance statement for instances DIP1, DIP2 and LED1 are : inst "DIP1" "IBUF", placed RIOIS_X17Y1 P78 , inst "DIP2" "IBUF", placed BIOIS_X16Y0 P72, inst "LED1" "IOB", placed RIOIS_X17Y1 P76 respectively. The instance view shown in Fig. 14

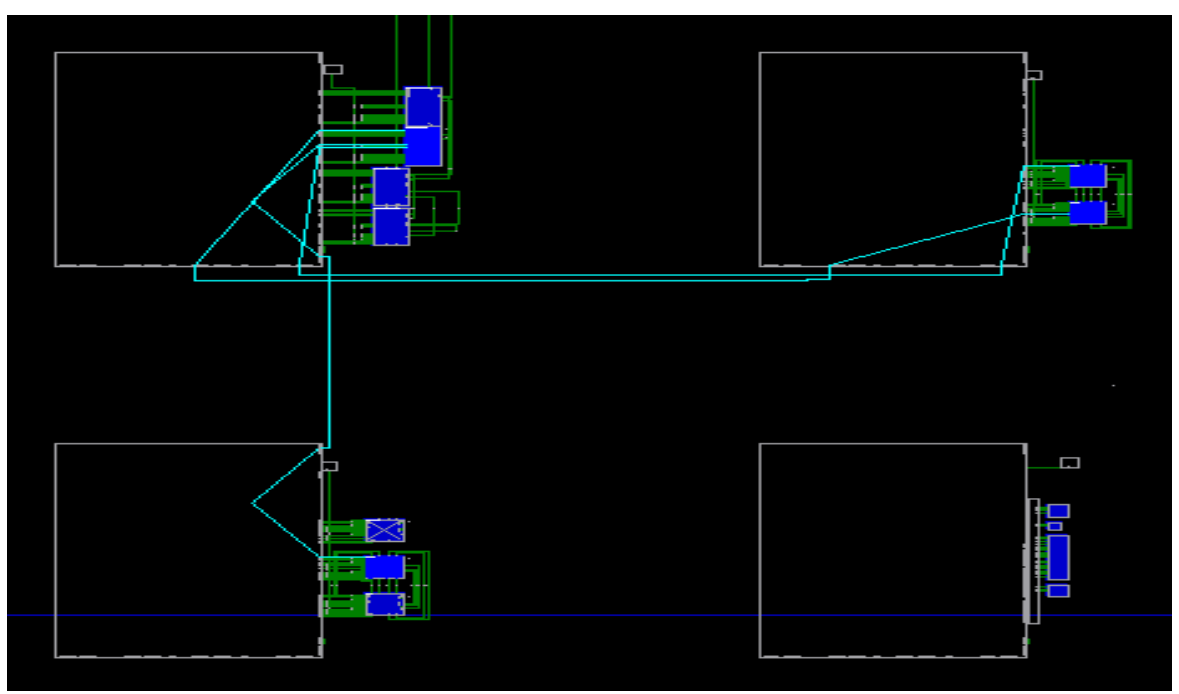

Fig. 13 (a) Default Design 


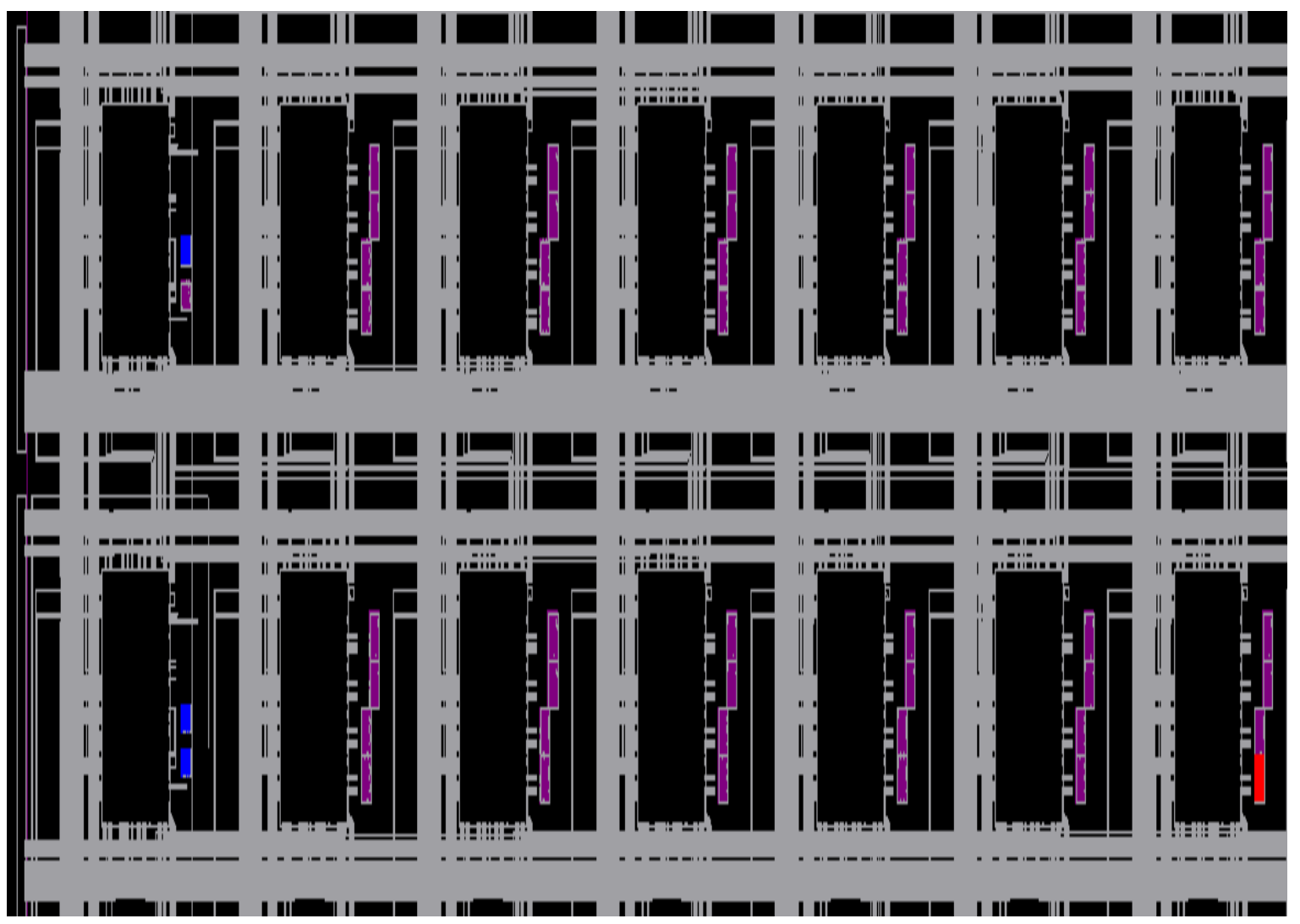

Fig. 13 (b) Design with Const_4

Fig. 13 The routing structure

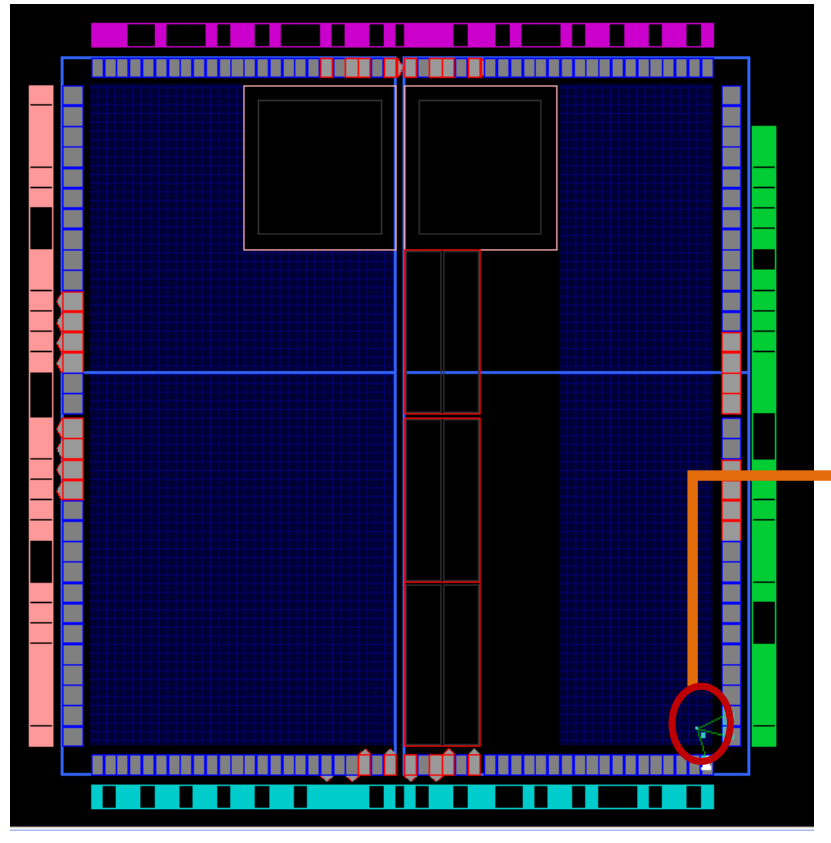

Fig. 14 (a) Location on FPGA layout

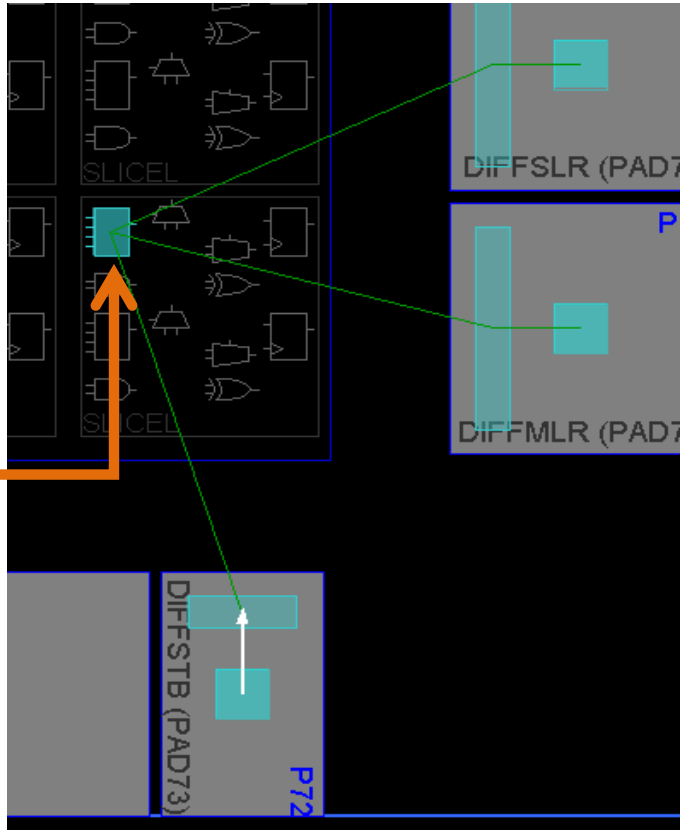

Fig. 14 (b) Zoom view of design

Fig. 14 Location of default design on FPGA 
The detail view of SLICE_X23Y0 as viewed in FPGA editor is depicted Fig. 15. Likewise view for other instances generated and routing of nets related with XDL file obtained in listing 2.

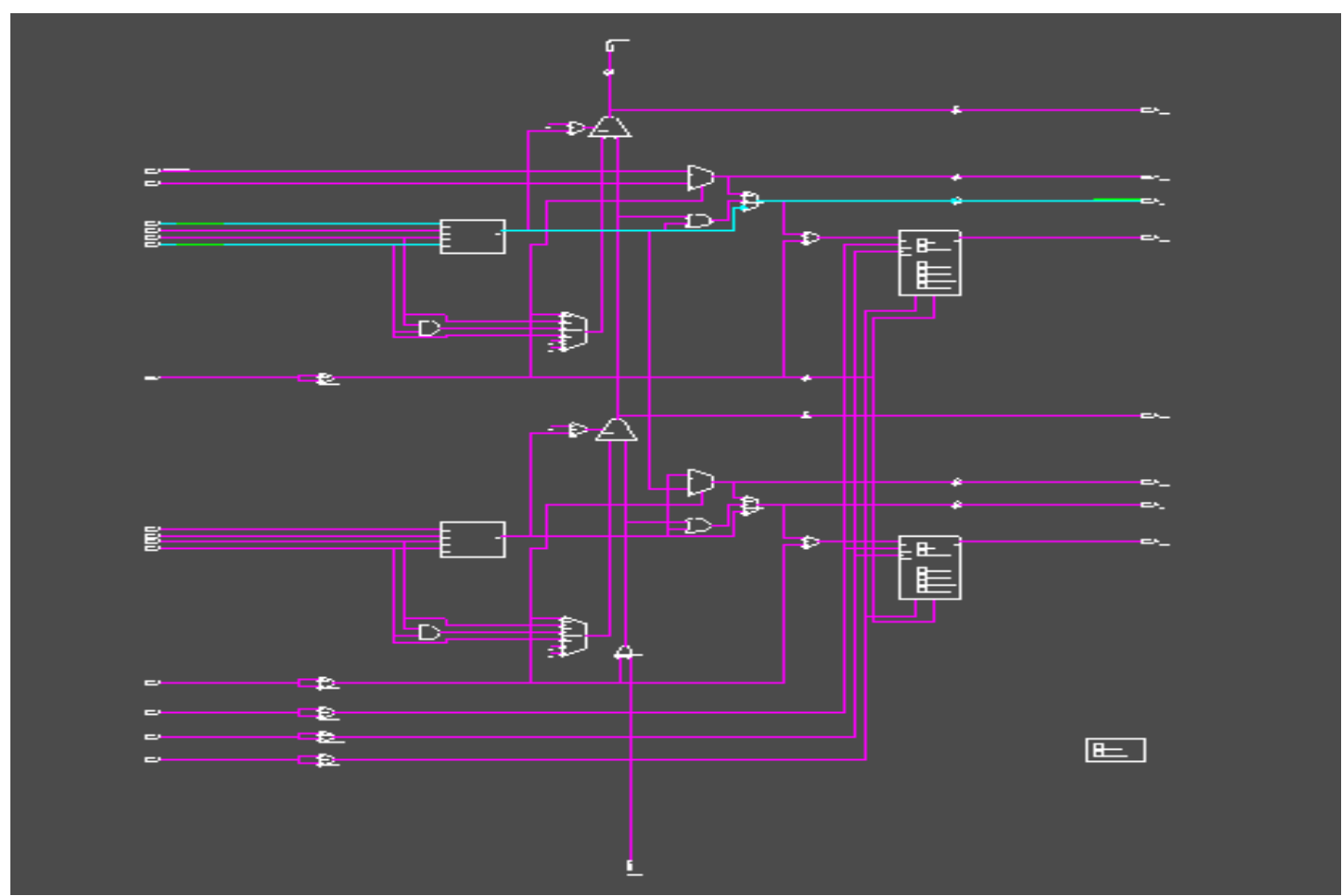

Fig. 15 LUT mapped on slice

\section{CONCLUSION}

A simplified straight approach to map FPGAs resources to its HDL description is demonstrated in this paper. We found that information in XDL file derived from NCD file is in human readable form and helps to develop the understanding of FPGA design layout. Xilinx ISE Design suite and PlanAhead tool played vital role in analysis. Though we are able to locate FPGA design location vertically with addresses information in FAR_MAJ register, configuration information pointed by FAR_MIN address register is yet not decoded for Spartan-3A FPGA by our approach. We observed that as we move any design from bottom-left position to topleft position to top-right position to top-bottom position, the configuration information in bitstream goes away and away form starting position of bitstream file. The addition of "Match Pattern" and the "Mismatch Pattern" in configuration bitstream file equals 368 for all design under study.

\section{REFERENCES}

[1] J.-B. Note and Eric Rannaud, "From the bitstream to the netlist", in Proceedings of the 2008 ACM/SIGDA 16th Annual International Symposium on Field-Programmable Gate Arrays, FPGA 2008 (Monterey, California), pp. 264-264, February 24-26, 2008,

[2] C. Beckhoff, D. Koch, and J. Torresen, "The xilinx design language (xdl): Tutorial and use cases," in Reconfigurable Communicationcentric Systems-on-Chip (ReCoSoC), 6th International Workshop, pp. 1-8, 2011

[3] Z. Ding, Q.Wu, Y. Zhang, and L. Zhu, "Deriving an NCD file from an FPGA bitstream: Methodology, architecture and evaluation", Microprocessors and Microsystems, vol 37, pp. 299-312, May 2013,

[4] Joushua Vasquez, “Reverse Engineering Lattice’s ICE40 FPGA Bitstream,” https://hackaday.com/2015/03/29/reverse-engineeringlattices-ice40-fpga-bitstream/

[5] F. Benz, A. Seffrin, and S. Huss, "Bil: A tool-chain for bitstream reverse-engineering,", in Field Programmable Logic and Applications (FPL), 22nd International Conference, 2012, pp. 735-738.

[6] Vaughn Betz and Jonathan Rose, "VPR: A new packing, placement and routing tool for FPGA research,” International Workshop on Field Programmable Logic and Applications, 1997.

[7] Casey J Morford, "BitMaT - Bitstream Manipulation Tool for Xilinx FPGAs," Master thesis. December $15^{\text {th }}$, 2005 Bradley Department of Electrical and Computer Engineering Blacksburg, Virginia.

[8] Khoa Dang Pham, Edson Horta and Dirk Koch, "BITMAN: A Tool and API for FPGA Bitstream Manipulations," Design, Automation \& Test in Europe Conference \& Exhibition (DATE), 27-31 March 2017.

[9] Adam Megacz, “A Library and Platform for FPGA Bitstream Manipulation,” 15th Annual IEEE Symposium on Field-Programmable Custom Computing Machines (FCCM 2007), 23-25 April 2006, PP 45-54.

[10] R. Soni, N. Steiner, and M. French, "Open-source bitstream generation," in Field-Programmable Custom Computing Machines”(FCCM), IEEE 21st Annual International Symposium , 2013, pp. 105-112.

[11] N. Steiner, A. Wood, H. Shojaei, J. Couch, P. Athanas, and M. French, "Torc: Towards an open-source tool flow", 19th International Symposium on Field-Programmable Gate Arrays (FPGA 2011), February 27-March 1, 2011. 
[12] Edson L. Horta, John W. Lockwood, "PARBIT: A Tool to transform bitfiles to implement partial reconfiguration of Field Programmable Gate Arrays (FPGAs),” Report Number: WUCS-01-13, Computer Science and Engineering, Washington University in St. Louis, 2001.

[13] C. Lavin, M. Padilla, P. Lundrigan, B. Nelson, and B. Hutchings, "Rapid prototyping tools for FPGA designs: RapidSmith," International Conference on Field-Programmable Technology (FPT'10), December 2010.

[14] Xilinx, “Command Line Tool User Guide, UG628 (v 14.7), October 2, 2013,” https://www.xilinx.com/support/documentation/sw_manuals/xilinx14_7/devref.pdf

[15] Xilinx, “ISE Design Suite 14: Release Notes, Installation, and Licensing.UG631 (v14.7) October 2, 2013,” https://www.xilinx.com/support/documentation/sw_manuals/xilinx14_7/irn.pdf

[16] Xilinx, "FPGA Editor Guide - 2.1i,"

http://ebook.pldworld.com/_semiconductors/Xilinx/Foundation/ISE/3.3i/Documentation/fpedit.pdf

[17] Xilinx, "Spartan-3 Generation Configuration User Guide (UG332 (v1.7) January 27, 2015 2.1)," https://www.xilinx.com/support/documentation/user_guides/ug332.pdf. 